Review Article

\title{
Precision Detection Technology: Equipping Precision Oncology with Wings
}

\author{
Rilan Bai, Zheng Lv, Xiao Chen, Hanfei Guo, Ling Bai, Huimin Tian, Wei Li $\mathbb{D}$, \\ and Jiuwei Cui iiD
}

Cancer Center, The First Hospital of Jilin University, Changchun, Jilin 130021, China

Correspondence should be addressed to Jiuwei Cui; cuijw@jlu.edu.cn

Received 10 December 2019; Accepted 23 June 2020; Published 9 July 2020

Academic Editor: Guido Bocci

Copyright (c) 2020 Rilan Bai et al. This is an open access article distributed under the Creative Commons Attribution License, which permits unrestricted use, distribution, and reproduction in any medium, provided the original work is properly cited.

\begin{abstract}
In recent years, precision medical detection techniques experienced a rapid transformation from low-throughput to highthroughput genomic sequencing, from multicell promiscuous detection to single-cell precision sequencing. The emergence of liquid biopsy technology has compensated for the many limitations of tissue biopsy, leading to a tremendous transformation in precision detection. Precision detection techniques contribute to monitoring disease development more closely, evaluating therapeutic effects more scientifically, and developing new targets and new drugs. In the future, the role of precision detection and the joint detection in epigenetics, rare gene detection, individualized targeted therapy, and multigene targeted drug combination therapy should be extensively explored. This article reviews the changes in precision medical detection technology in the era of precision medicine, as well as the development, clinical application, and future challenges of liquid biopsy.
\end{abstract}

\section{Introduction}

Precision medicine development should be based on accurate detection and should take into account various factors, including genetic and environmental susceptibly factors including living habits in order to identify disease etiology, pathogenesis, and therapeutic targets. The aim of precision medicine is to eventually improve the benefits of disease in diagnosis, treatment, and prevention, as well as achieve optimal personalized precision treatment [1]. The era of precision medicine has brought tremendous changes for the diagnosis and treatment of cancer. The discovery of tumor-driven genes has led to the development of targeted therapeutic drugs that have promoted individualized precision treatment [2] but have also brought challenges to precision medical detection technology. In recent years, clinical demands have gradually driven the development and application of precision detection techniques and promoted the emergence of new technologies, new targets, and new drugs in these times of precision medicine.

\section{The Development of Precision Sequencing Technology}

Given that different pathological tumor types have different gene mutations, precision medical detection technology has been revolutionized from the detection of mutations at a single site to multiple sites simultaneously, from lowthroughput to high-throughput sequencing and from multicell hybrid detection to single-cell precision detection. It has achieved the simultaneous detection of multitarget detection of genes, which promotes the development of precision medicine.

2.1. Low-Throughput Genomic Sequencing to High-Throughput Genomic Sequencing. The first-generation sequencing technology (Sanger sequencing) was difficult to universalize as it was expensive and time consuming. The constant demand of the clinic led to the emergence of next-generation sequencing (NGS) that can obtain all the genetic information and provide a "real-time" snapshot of the tumor genetic 
landscape by combining polymerase chain reaction (PCR) with fluorescent labeling imaging technology [3]. Currently, many NGS-based tumor gene panels have been used in clinical practice, such as FoundationOne CDx detecting 324 gene mutations and MSK-IMPACT detecting 468 gene mutations approved by the U.S. Food and Drug Administration (FDA). Although there are many advantages, NGS is technically limited because the reading length is short, which would cause regional assembly errors of high complexity of the genome, increased sequencing gap area, and difficulty in detection of low abundance. Not only that, NGS would introduce many potential deviations and errors during data reading and sequence assembly due to the need of amplification and segmentation during the process of database construction [4].

The third-generation single-molecule sequencing technology has gradually developed [5]. It compensates for the disadvantage of shorter read length of the second-generation sequencing and does not require PCR amplification, allowing rapid sequencing splicing of whole genome sequences (WGS), avoiding the above sequencing deviations and being more advantageous in sequencing cost and time. This technology can also obtain both genetic and epigenetic information at the single nucleotide level from DNA, providing important technical support for the in-depth analysis of the epigenetic mechanisms of different metabolic processes in organisms. Nevertheless, the analysis of epigenetic information at the single nucleotide level is not the exclusive characteristic of single-molecule sequencing. In tumor detection, single-molecule sequencing technology can detect not only genetic mutations but also epigenetic modification sites and the presence of long-chain noncoding RNAs, allowing tumor classification and grading $[6,7]$. RNA-sequencing (RNA-seq) is a relatively new application of NGS that is gradually replacing microarrays as the preferred technology to analyze transcripts. RNA-seq can quantify gene expression, detect new transcripts, and analyze small noncoding RNAs that are not translated into proteins $[8,9]$. Large RNA-seq datasets using whole-blood RNA-seq combined with variant and phenotypic-related genes have practical value in identifying abnormal expression, splicing, and alternative splicing events in rare disease candidate genes [10].

2.2. Multicell Hybrid Detection to Single-Cell Precision Detection. Traditional genetic testing is based on mixed-cell DNA samples. The results of this analysis represent the characteristics of most cells in the tissue or the average of the whole sample, making it impossible to characterize the intercellular differences and interpret the growth and development mechanism of the organism and the features of a small number of special cells. In recent years, single-cell sequencing technology, based on high-throughput sequencing analysis of the genome, transcriptome, and epigenetics of single cells, has allowed to analyze tumor heterogeneity and clonal evolution and to reveal the molecular mechanisms of tumor chemotherapy resistance [11]. Single-cell sequencing technology can detect microgenes and rare noncoding RNAs and can reveal the evolution of individual cells at specific times that helps to understand the occurrence and evolution of tissues or organisms at the individual level [11-13].

Single-cell sequencing allows the construction of immune cell maps and the study of the tumor immune microenvironment [14-16]. Guo et al. [15] used single-cell sequencing to study the subpopulation, baseline of lineage, and functional status of tumor infiltrating lymphocytes (TILs) and designed the non-small cell lung cancer (NSCLC) T-lymphocyte map. It includes a single-cell data acquisition process and immune profiles such as T-cell subset classification, state transitions between subpopulations, and heterogeneity of regulatory T-cells. In addition, single-cell sequencing can also reveal cellular spatial localization. Keren et al. [17] obtained single-cell level information by isotope labeled proteins, named multiplexed ion beam imaging by time of flight (MIBI-TOF). This technology allows to systematically understand the spatial distribution characteristics of breast cancer cells and different types of immune cells to accurately visualize the cell distribution characteristics of different patients and to further evaluate the potential efficacy of immunotherapy and the prognosis of a disease.

\section{Revolutionary Changes Brought by Liquid Biopsy Technology for Precision Medical Testing}

Due to the continuity of spatiotemporal and dynamic evolution, tumor cells and genomic variation appear highly heterogeneous. Tumor heterogeneity is broadly classified into inter- and intratumor heterogeneity $[18,19]$. The high heterogeneity and rapid evolution of tumors is the biggest obstacle to accurate detection and diagnosis of tumors $[20,21]$. Sampling area deviation may occur during tissue biopsy, that is, one regional tumor feature cannot represent the entire tumor, and continuous extensive tissue sampling of primary and metastatic lesions is difficult to achieve. In addition, tissue biopsy is limited to the characteristics of invasive and static detection, so it is urgent to find a more comprehensive testing method that reflects the characteristics and dynamic changes of tumors.

In recent years, the emergence of liquid biopsy technology seems to enable the precision detection from static to dynamic. It allows to repeatedly and dynamically monitor the evolution of tumors over time and become the most powerful tool for studying intratumor heterogeneity by simple noninvasive blood sampling and analysis [22, 23]. This technology uses PCR or high-throughput sequencing to detect circulating tumor DNA (ctDNA), circulating tumor cells (CTCs), extracellular vesicles (EVs), and circulating tumor RNA (ctRNA) released from the primary tumor and metastatic sites into the bloodstream [24, 25]. Clinically, liquid biopsy can achieve comprehensive and dynamic monitoring of tumor markers, so it has significant clinical applications in tumor screening and early detection, realtime monitoring of tumor treatment, researching new treatment targets and drug resistance mechanisms, and 
predicting risks of metastatic recurrence. Although some studies have confirmed the potential of liquid biopsy to change the mode of cancer treatment, future investigations are needed to fully evaluate its role in tumor detection and treatment [26].

\subsection{The Detection and Clinical Application of ctDNA}

3.1.1. The Development of ctDNA Detection Technology. Quantitative information can be obtained from the measurement of the mutant allele fraction (MAF), which is a reflection of the tumor burden. It is used to detect minimal residual disease (MRD) and occult metastases; it is also a high-sensitivity, high-specificity predictor in monitoring therapeutic effectiveness and relapse. Qualitative information can be sourced through the profiling of mutations, amplifications, deletions, and translocations in ctDNA. This allows the identification of genetic alterations associated with response, hence supporting decision making for personalized management [25]. In recent years, a variety of detection methods of ctDNA epidermal growth factor receptor (EGFR)-mutation have been developed, including the amplification refractory mutation system (ARMS), droplet digital PCR (ddPCR), and NGS [27]. ARMS is an economical and rapid method approved by the FDA for clinical testing, but its sensitivity is only about 50\% [28-30]. ddPCR and NGS have significantly improved detection accuracy, but the operation process is too complicated and expensive and is therefore not widely used in clinical practice. The SuperARMS, an improved method of ARMS, was recently proposed with enhanced sensitivity and a simplified operation process. China FDA (CFDA) has approved it to detect EGFR mutation status in NSCLC patients to screen for those suitable for targeted drugs [31]. Li et al. calculated the clinical efficacy of the SuperARMS EGFR test using EGFR mutation status in tumor tissue as a standard reference. The results showed that EGFR mutations were detected in $45.9 \%$ (50/ 109) of plasma samples and $56.9 \%(62 / 109)$ of matched tumor tissue samples and verified the sensitivity (82\%), specificity $(100 \%)$, positive predictive value $(100 \%)$, and negative predictive value (81.4\%) of SuperARMS [31]. The AURA 17 expansion study (NCT01802632) used Cobas, SuperARMS, and ddPCR to detect ctDNA EGFR gene mutations in NSCLC cases of the Asia-Pacific population. The results showed the positive detection rates of the three blood tests compared to the tissue test results, which were $42 \%, 49 \%$, and $56 \%$, respectively. The objective response rate (ORR) of osimertinib in T790M-positive patients was $56 \%$, $64 \%$, and $56 \%$, respectively. This study confirms the accuracy of SuperARMS in the prediction and guidance of clinical medication, proposing it as the optimal choice for clinical blood ctDNA testing.

In addition to single-gene detection, ctDNA-based multigene analysis techniques, such as blood TMB (bTMB) and microsatellite instability (MSI) tests, show more convenient, fast, and dynamic characteristics compared with histological detection [32]. Recent results from POPLAR and OAK clinical studies have shown that bTMB can predict the efficacy of immunotherapy and that patients are more likely to benefit from immunotherapy as the cutoff value of bTMB increases [33, 34].

\subsubsection{The Clinical Applications of ctDNA}

(1) Early Diagnosis of Tumor. Early screening of tumors through screening tests is a key step in early diagnosis and treatment of tumor. Early screening tests should be highly sensitive, specific, and cost-effective. However, a concern in early detection is the predictive value of single or small sets of mutations, since cancer-associated mutations can also be found in the plasma of healthy individuals as a result of clonal hematopoiesis [30]. One approach to overcome this challenge is the CancerSEEK platform recently proposed by Cohen et al. [35], which detects eight common cancer types by detecting rare mutations and tumor-derived protein biomarkers expected to be present in plasma ctDNA. The assay platform evaluates levels of eight proteins and the presence of mutations in 1933 different genomic locations, using rigorous statistical methods to ensure the accuracy of the test, such as logistic regression algorithm, 10 iterations of 10 -fold cross-validations. The results showed that a median sensitivity of CancerSEEK tests was $70 \%$ of the eight cancer types, ranging from $69 \%$ to $98 \%$ for the detection of five cancer types (ovary, liver, stomach, pancreas, and esophagus), and the specificity was more than $99 \%$. However, this study contains some limitations, and the sensitivity and false positive rate of the test require further research to verify.

(2) Guidance for Tumor Treatment and Prediction of Therapeutic Efficacy. The phase II BENEFIT study [36] proposed that dynamic monitoring of ctDNA changes can predict tumor treatment efficacy. The results showed that progression-free survival (PFS) was significantly longer in patients whose peripheral blood ctDNA EGFR mutations disappeared after 8 weeks of receiving first-line gefitinib. In addition, dynamic monitoring of ctDNA in immunotherapy can help to follow tumor residual disease, identify pseudoprogression, and predict the efficacy of immunotherapy. Lee et al. [37] confirmed that the changes of the ctDNA spectrum after 12 weeks of treatment could accurately distinguish between pseudoprogression and true disease progression. Using ctDNA abundance to identify pseudoprogression showed $90 \%$ sensitivity and $100 \%$ specificity, while the lactate dehydrogenase (LDH) profile showed $60 \%$ sensitivity and $89 \%$ specificity. They also verified that there was a significant difference in 1-year survival rates between patients with benign and malignant ctDNA changes (82\% and 39\%) [37].

(3) Prediction of Tumor Recurrence and Metastasis. Coombes et al. [38] performed plasma ctDNA sequencing (ultradeep sequencing) on 49 patients with postoperative or adjuvant breast cancer every 6 months and monitored CT imaging, liver function tests (LFT), and CA15-3 levels. The results showed that the specificity of breast cancer recurrence by ctDNA was $100 \%$ and the sensitivity was $89 \%$, which was more accurate 
than the other three traditional detection methods. Another study confirmed that the continuous monitoring of ctDNA levels using the Safe-SeqS technique during postoperative follow-up in patients with stage III nonmetastatic CRC could identify disease recurrence prior to routine imaging studies [39]. In this study cohort, all 8 patients with recurrence had positive ctDNA during follow-up (100\%, 95\% CI, 63\%-100\%), whereas 5 of 8 patients with recurrence had positive CEA levels (>5 $\mu \mathrm{g} / \mathrm{L})(63 \%$; 95\% CI, 24\%-91\%). Another study used NGS to detect mutations in serum samples at 1 and 3 months from 53 patients with acute myeloid leukemia and myelodysplastic syndrome (AML/MDS) receiving allogeneic hematopoietic stem cell transplantation (alloSCT), and at least one APCR was performed in each case. The results showed persistent mutations in the bone marrow at 1 and 3 months [mutation persistence (MP): MP1 and MP3], and matched serum [ctDNA persistence (CP): CP1 and CP3] after alloSCT was associated with a higher 3-year cumulative recurrence rate, confirming the effectiveness of ctDNA in predicting the risk of disease recurrence [40].

\subsubsection{Methylation Detection in ctDNA Broadens the Field of} Precision Medical Research. DNA methylation is one of the earliest epigenetic changes observed in tumor development, and its abnormal pattern is highly specific in different tumor tissues [41, 42]. Previous studies of epigenomics mainly relied on microarray technology, but the advent of NGS has largely facilitated the study of high-throughput data to detect epigenetic changes across the genome at single nucleotide resolution. Detection techniques for gene methylation include PCR, whole-genome methylation sequencing, and $450 \mathrm{~K}$ methylation chips. Clinically, ctDNA methylation can reflect the hypermethylation characteristics of the promoter region of a gene, and the dual signals of tumor markers and tissuespecific methylation patterns can be used to detect and localize tumors. Additionally, detection of ctDNA methylation can be used for early tumor diagnosis and assessment of prognosis, as it can reflect tumor load changes [43, 44]. The most widely used lung cancer-specific gene methylation detection kit is the human SHOX2 and RASSF1A gene methylation DNA detection kit [45], which could assist in the early diagnosis of lung cancer and in the differentiation between benign and malignant pulmonary nodules. Additionally, together with a cytological test, it can effectively improve the sensitivity and specificity of lung cancer detection. Studies have shown that the combined detection of multiple gene methylation markers can help to improve the sensitivity and specificity of lung cancer diagnosis, reaching $71.5 \%-83.2 \%$ and $90 \%-97.4 \%$, respectively [46]. A large-scale clinical data analysis study proposed methylation models for early screening, risk assessment, and prognosis monitoring of liver cancer, including a comprehensive diagnostic model (cdscore) and a comprehensive prognostic model (cp-score), and verified its potential role in tumor monitoring [47].

3.2. The Detection and Clinical Application of CTCs. CTCs are defined as cells that shed from the primary tumor site or from metastases and enter the blood circulation through the vascular or lymphatic system [48]. Their presence is considered the basis for tumor metastasis development. CTCs undergo vascular leakage by epithelial-mesenchymal transition (EMT) [49] or passive shedding of primary tumors. The latter mechanism is supported by the presence of CTCs aggregates or circulating tumor microemboli (CTM) in the blood [50]. In clinical practice, CTCs can be isolated and enriched through their biological and physical properties such as size, density, and charge or through specific molecules on their surface based on antigen-antibody recognition. Once identified, their nucleic acid can be analyzed by cytochemical staining, fluorescence in situ hybridization, PCR, and sequencing, among others. At present, the only detection technology of CTCs approved by the FDA for clinical use is the Cell Search system [51], which defines CTCs as epithelial cell adhesion molecule (EpCAM)+/ cytokeratin (CK)+/4,6-diamidino-2-phenylindole (DAPI)+/ CD45+. CTCs can provide clinical quantitative information through cell counting and qualitative information based on single-cell genome, transcriptomics, and proteomic analysis.

CTCs can reflect the tumor tissue and can help carry out pathological diagnoses, molecular sequencing, and disease monitoring, as well as providing treatment decision making and prognosis information for the clinic through noninvasive dynamic monitoring [52]. Scher et al. [53] performed blood CTCs and immunofluorescence staining on 142 patients treated with ARS inhibitors or taxanes and detected the expression of androgen receptor splice variant 7 (ARV7) in CTCs. The overall survival (OS) of AR-V7-positive patients treated with taxanes was higher than in those treated with an androgen receptor signaling (ARS) inhibitor (median OS 14.3 vs. 7.3 months, $p=0.25$ ), while AR-V7-negative patients treated with ARS inhibitors had a higher OS than those treated with taxanes. It is suggested that future the research studies of CTCs should look into the analysis of macromolecules expressed by CTCs and the design of therapeutic measures to target them.

3.3. The Detection and Clinical Application of EVs. EVs are lipid vesicles secreted by cells that carry various proteins and nucleic acids, which can provide tumor-related information $[54,55]$. Studies have shown that PD-L1riched exosomes secreted by tumor cells can enter the peripheral blood of the body and inhibit the T-cells outside of the tumor microenvironment. Therefore, detecting EVs could help understand the mechanism of tumor immune escape and consequently develop targeted therapies to overcome immunosuppression [56]. Liquid biopsy technology of exosomes, known as ExoDx Prostate IntelliScore (EPI), has been approved by the FDA for the detection and prognosis assessment of prostate cancer. The study also showed that the Friend leukemia virus integration 1 (FLI1) exon cyclic RNA is a new carcinogenic driver that promotes tumor metastasis via the miR584-ROCK1 pathway. The content of FECR 1 in serum exosomes is related to the therapeutic efficacy and prognosis of patients with small cell lung cancer and could therefore be used as a new marker for efficacy monitoring and prognosis evaluation $[57,58]$. 
3.4. Combined Detection of Liquid Biopsy Expands the Application of Precision Medicine. Genomics combined with transcriptomics or proteomic analysis extends the application of precision medicine to provide the best treatment options for cancer. The WINTHER trial [59] recommends precise treatments for refractory tumors by integrating DNA and RNA gene expression profiles. Transcriptomics significantly increased the proportion of patients receiving matched treatment, and $22.4 \%$ of patients that received the recommended treatment had a PFS ratio of previously treated $>1.5$. CancerSEEK, a blood test, can detect eight common cancer types through assessment of the levels of eight circulating proteins and sixteen mutations in ctDNA, with a specificity of over $99 \%$ and a median sensitivity of $43 \%$ in stage I tumors, $73 \%$ in stage II, and $78 \%$ in stage III [35]. Krug et al. [60] collected tumor tissues and blood samples from 84 patients who participated in the TIGER-X test (NCT01526928) and examined ctDNA and ctRNA using the exoNA [detection of RNA (exoRNA) in extracellular vesicle exosomes combined with ctDNA based on NGS] method and the BEAMing (ddPCR) method. The results showed that the exoNA assay had sensitivity of $98 \%$ for detecting EGFR mutations and $90 \%$ for EGFR T790M mutations, while the sensitivity of the BEAMing method was $82 \%$ for EGFR mutations and $84 \%$ for EGFR T790M mutations, indicating the advantage of multidimensional combined detection of blood biomarkers.

\subsection{The Clinical Limitations of Liquid Biopsy Technology.} At present, there are several technical limitations in liquid biopsy. Firstly, plasma can be used as a substitute biomaterial instead of tissue to detect tumor information, but its detection sensitivity still needs to be optimized. In clinical practice, it is necessary to fully evaluate its false negative rate, and repeated liquid biopsies or tissue repeat tests should be obtained from patients with negative plasma tests. Secondly, clinical practice of ctDNA analysis lacks a standardized protocol for preanalytical sample preparation and purification. The current analytical testing procedures are complex and may lead to ctDNA degradation and cytolysis. The analytical platform for rapid purification from the blood is a difficult problem to be solved in the future. In addition, challenges in detecting CTCs include extreme rarity, fragility, and physical and phenotypic heterogeneity. Finally, the predictive value of single or small sets of mutations in early detection is limited, and joint detection methods, such as the combination of genomics and transcriptomics or proteomics, may be a new technique to improve tumorspecific detection, like the CancerSEEK platform. Although some studies have confirmed the potential of liquid biopsy to change the mode of cancer treatment, a lot of research is still required in the future to fully define its role in tumor diagnosis, monitoring, and prognosis.

\section{Summary and Future Directions}

Accurate detection techniques promote the development of individualized cancer diagnosis and treatment in the era of precision medicine. At the same time, the demand for clinical precision diagnosis and treatment can further drive the improvement and application of precision detection technology. Precision medicine relies on a thorough understanding of tumor biology. New technologies allow us to map tumors, identify key carcinogenic drivers, and formulate treatments that specifically target tumor cells to achieve clinical benefits. In recent years, precision medical detection techniques promoted the emergence and development of new technologies, new targets, and new drugs in the times of precision medicine. This has accelerated research progress and improved the understanding of disease risk mechanisms and predictions of optimal treatment options for multiple diseases.

At present, there are still many challenges in precision detection techniques and platform construction. Firstly, the results obtained from different NGS-based technologies are inconsistent, which may relate to sample preprocessing, NGS platform, and algorithm differences. Secondly, the sensitivity and accuracy of liquid biopsy need to be verified by further evidence, and it cannot yet be used for early screening and diagnosis of tumors [61]. Thirdly, the cost of single-cell sequencing technology, the need for freshly isolated cells, and the high level of laboratory requirements limit its extensive clinical application. In addition, studies have shown that therapies targeting the same gene mutation have different effects in different tumor types, which may be related to differences in signal transduction and intertumor heterogeneity [62]. The development of precision detection techniques contributes to explore its internal mechanisms and more accurately predict individual patient responses to treatment and achieve individualized precision medical treatment.

In the future, the role of precision detection and combined detection in epigenetics, rare gene detection, individualized targeted therapy, and multigene targeted drug combination therapy should be extensively explored [63]. The accumulation of large samples and big data and the development of cloud computing and artificial intelligence are expected to accelerate scientific research progress, to promote the development of medical science and technology, and to expand the health industry. Precision detection techniques make it possible to understand the molecular characteristics of each patient and the information provided by mutations to develop personalized diagnostic or treatment programs. Also, precision detection techniques contribute to monitoring disease development more closely, evaluating therapeutic effects more scientifically, and fully comprehending the requirements of precision medicine.

\section{Conflicts of Interest}

The authors have no conflicts of interest to declare.

\section{Acknowledgments}

The authors thank Rilan Bai, Hanfei Guo, and Jiuwei Cui for their article "Development status and clinical applications of precision detection technology in the times of precision 
oncology" in "Chinese Journal of Cancer Biotherapy," which gave them the basis for preliminary investigation. This study was supported by the National Key R\&D Program of China (no. 2016YFC1303800), Innovation Project of Health and Technology in Jilin Province (no. 2017J064), 13th Five-Year Science and Technology Project of Jilin Provincial Education Department (no. JJKH20190020KJ), Project of Department of Science and Technology of Jilin Province (no. 20180101009JC), Special Project of Development and Reform Commission in Jilin Province (no. 2017C022), and Key Laboratory Construction Project of Department of Science and Technology of Jilin Province (no. 20170622011JC).

\section{References}

[1] K. T. Schmidt, C. H. Chau, D. K. Price, and W. D. Figg, "Precision oncology medicine: the clinical relevance of patient-specific biomarkers used to optimize cancer treatment," The Journal of Clinical Pharmacology, vol. 56, no. 12, pp. 1484-1499, 2016.

[2] R. S. Herbst, D. Morgensztern, and C. Boshoff, "The biology and management of non-small cell lung cancer," Nature, vol. 553, no. 7689, pp. 446-454, 2018.

[3] C. Paolillo, E. Londin, and P. Fortina, "Next generation sequencing in cancer: opportunities and challenges for precision cancer medicine," Scandinavian Journal of Clinical and Laboratory Investigation, vol. 76, no. 245, pp. S84-S91, 2016.

[4] H. P. J. Buermans and J. T. den Dunnen, "Next generation sequencing technology: advances and applications," Biochimica et Biophysica Acta (BBA)-Molecular Basis of Disease, vol. 1842, no. 10, pp. 1932-1941, 2014.

[5] V. A. Arboleda and R. R. Xian, "An overview of DNA analytical methods," Methods in Molecular Biology, vol. 1897, pp. 385-402, 2019.

[6] A. Ameur, W. P. Kloosterman, and M. S. Hestand, "Singlemolecule sequencing: towards clinical applications," Trends in Biotechnology, vol. 37, no. 1, pp. 72-85, 2019.

[7] P. Jenjaroenpun, T. Wongsurawat, R. Pereira et al., "Complete genomic and transcriptional landscape analysis using thirdgeneration sequencing: a case study of Saccharomyces cerevisiae CEN.PK113-7D," Nucleic Acids Research, vol. 46, no. 7, p. e38, 2018.

[8] L. D. Goldstein, Y. Cao, G. Pau et al., "Prediction and quantification of splice events from RNA-seq data," PLoS One, vol. 11, no. 5, Article ID e0156132, 2016.

[9] T. Steijger, J. F. Abril, J. F. Abril et al., "Assessment of transcript reconstruction methods for RNA-seq," Nature Methods, vol. 10, no. 12, pp. 1177-1184, 2013.

[10] L. Frésard, C. Smail, C. Smail et al., "Identification of raredisease genes using blood transcriptome sequencing and large control cohorts," Nature Medicine, vol. 25, no. 6, pp. 911-919, 2019.

[11] J. E. Wiedmeier, P. Noel, W. Lin, D. D. Von Hoff, and H. Han, "Single-cell sequencing in precision medicine," Precision Medicine in Cancer Therapy, vol. 178, pp. 237-252, 2019.

[12] W. Zhu, X.-Y. Zhang, S. L. Marjani et al., "Next-generation molecular diagnosis: single-cell sequencing from bench to bedside," Cellular and Molecular Life Sciences, vol. 74, no. 5, pp. 869-880, 2017.

[13] X. Zhang, S. L. Marjani, Z. Hu, S. M. Weissman, X. Pan, and $\mathrm{S}$. Wu, "Single-cell sequencing for precise cancer research: progress and prospects," Cancer Research, vol. 76, no. 6, pp. 1305-1312, 2016.
[14] H. Li, A. M. van der Leun, I. Yofe et al., "Dysfunctional CD8 $\mathrm{T}$ cells form a proliferative, dynamically regulated compartment within human melanoma," Cell, vol. 176, no. 4, pp. 775-789, 2019.

[15] X. Guo, Y. Zhang, L. Zheng et al., "Global characterization of $\mathrm{T}$ cells in non-small-cell lung cancer by single-cell sequencing," Nature Medicine, vol. 24, no. 7, pp. 978-985, 2018.

[16] E. Azizi, A. J. Carr, G. Plitas et al., "Single-cell map of diverse immune phenotypes in the breast tumor microenvironment," Cell, vol. 174, no. 5, pp. 1293-1308, 2018.

[17] L. Keren, M. Bosse, D. Marquez et al., "A structured tumorimmune microenvironment in triple negative breast cancer revealed by multiplexed ion beam imaging," Cell, vol. 174, no. 6, pp. 1373-1387, 2018.

[18] S. Turajlic, A. Sottoriva, T. Graham, and C. Swanton, "Resolving genetic heterogeneity in cancer," Nature Reviews Genetics, vol. 20, no. 7, pp. 404-416, 2019.

[19] B. Vogelstein, N. Papadopoulos, V. E. Velculescu, S. Zhou, L. A. Diaz, and K. W. Kinzler, "Cancer genome landscapes," Science, vol. 339, no. 6127, pp. 1546-1558, 2013.

[20] M. Jamal-Hanjani, G. A. Wilson, N. McGranahan et al., "Tracking the evolution of non-small-cell lung cancer," New England Journal of Medicine, vol. 376, no. 22, pp. 2109-2121, 2017.

[21] M. Gerlinger, A. J. Rowan, S. Horswell et al., "Intratumor heterogeneity and branched evolution revealed by multiregion sequencing," The New England Journal of Medicine, vol. 366, no. 10, pp. 883-892, 2012.

[22] G. De Rubis, S. Rajeev Krishnan, and M. Bebawy, "Liquid biopsies in cancer diagnosis, monitoring, and prognosis," Trends in Pharmacological Sciences, vol. 40, no. 3, pp. 172-186, 2019.

[23] S. Mader and K. Pantel, "Liquid biopsy: current status and future perspectives," Oncology Research and Treatment, vol. 40, no. 7-8, pp. 404-408, 2017.

[24] G. De Rubis, S. R. Krishnan, and M. Bebawy, "Circulating tumor DNA-current state of play and future perspectives," Pharmacological Research, vol. 136, pp. 35-44, 2018.

[25] L. Cabel, C. Proudhon, E. Romano et al., "Clinical potential of circulating tumour DNA in patients receiving anticancer immunotherapy," Nature Reviews Clinical Oncology, vol. 15, no. 10, pp. 639-650, 2018.

[26] J. Wang, S. Chang, G. Li, and Y. Sun, "Application of liquid biopsy in precision medicine: opportunities and challenges," Frontiers of Medicine, vol. 11, no. 4, pp. 522-527, 2017.

[27] M. Postel, A. Roosen, P. Laurent-Puig, V. Taly, and S.-F. Wang-Renault, "Droplet-based digital PCR and next generation sequencing for monitoring circulating tumor DNA: a cancer diagnostic perspective," Expert Review of Molecular Diagnostics, vol. 18, no. 1, pp. 7-17, 2018.

[28] H. Xu, A. A. H. Baidoo, A. A. H. Baidoo et al., "A comparison of EGFR mutation status in tissue and plasma cell-free DNA detected by ADx-ARMS in advanced lung adenocarcinoma patients," Translational Lung Cancer Research, vol. 8, no. 2, pp. 135-143, 2019.

[29] X. Li, R. Ren, S. Ren et al., "Peripheral blood for epidermal growth factor receptor mutation detection in non-small cell lung cancer patients," Translational Oncology, vol. 7, no. 3, pp. 341-348, 2014.

[30] M. S. Hung, J. H. Lung, Y. C. Lin et al., "Comparative analysis of two methods for the detection of EGFR mutations in plasma circulating tumor DNA from lung adenocarcinoma patients," Cancers, vol. 11, no. 6, 2019. 
[31] Y. Li, H. Xu, S. Su et al., "Clinical validation of a highly sensitive assay to detect EGFR mutations in plasma cell-free DNA from patients with advanced lung adenocarcinoma," PLoS One, vol. 12, no. 8, Article ID e0183331, 2017.

[32] Stern M. H., Pierga J. Y., Bidard F. C..

[33] T. Mio, K. Keisuke, G. Kiyoshi et al., "Perivascular epithelioid cell tumor with SFPQ/PSF-TFE3 gene fusion in a patient with advanced neuroblastoma," American Journal of Surgical Pathology, vol. 33, no. 9, pp. 1416-1420, 2009.

[34] K. Byrne, G. Zanotti, P. Hallworth et al., "Real-world treatment patterns and outcomes of patients with stage IV squamous cell carcinoma of the head and neck," Future Oncology, vol. 15, no. 6, pp. 611-623, 2019.

[35] J. D. Cohen, L. Li, Y. Wang et al., "Detection and localization of surgically resectable cancers with a multi-analyte blood test," Science, vol. 359, no. 6378, pp. 926-930, 2018.

[36] Z. Wang, Y. Cheng, T. An et al., "Detection of EGFR mutations in plasma circulating tumour DNA as a selection criterion for first-line gefitinib treatment in patients with advanced lung adenocarcinoma (BENEFIT): a phase 2 , singlearm, multicentre clinical trial," The Lancet Respiratory Medicine, vol. 6, no. 9, pp. 681-690, 2018.

[37] J. H. Lee, G. V. Long, A. M. Menzies et al., "Association between circulating tumor DNA and pseudoprogression in patients with metastatic melanoma treated with anti-programmed cell death 1 antibodies," JAMA Oncology, vol. 4, no. 5, pp. 717-721, 2018.

[38] R. C. Coombes, K. Page, R. Salari et al., "Personalized detection of circulating tumor DNA antedates breast cancer metastatic recurrence," Clinical Cancer Research, vol. 25, no. 14, pp. 4255-4263, 2019.

[39] Y. Wang, L. Li, J. D. Cohen et al., "Prognostic potential of circulating tumor DNA measurement in postoperative surveillance of nonmetastatic colorectal cancer," JAMA Oncology, vol. 5, no. 8, pp. 1118-1123, 2019.

[40] S. Nakamura, K. Yokoyama, E. Shimizu et al., "Prognostic impact of circulating tumor DNA status post-allogeneic hematopoietic stem cell transplantation in AML and MDS," Blood, vol. 133, no. 25, pp. 2682-2695, 2019.

[41] S. Biswas and C. M. Rao, "Epigenetics in cancer: fundamentals and beyond," Pharmacology \& Therapeutics, vol. 173, pp. 118-134, 2017.

[42] K. Holm, J. Staaf, M. Lauss et al., "An integrated genomics analysis of epigenetic subtypes in human breast tumors links DNA methylation patterns to chromatin states in normal mammary cells," Breast Cancer Research, vol. 18, no. 1, p. 27, 2016.

[43] K. Ma, B. Cao, and M. Guo, "The detective, prognostic, and predictive value of DNA methylation in human esophageal squamous cell carcinoma," Clinical Epigenetics, vol. 8, p. 43, 2016.

[44] S. Guo, D. Diep, N. Plongthongkum, H.-L. Fung, K. Zhang, and K. Zhang, "Identification of methylation haplotype blocks aids in deconvolution of heterogeneous tissue samples and tumor tissue-of-origin mapping from plasma DNA," Nature Genetics, vol. 49, no. 4, pp. 635-642, 2017.

[45] C. Zhang, W. Yu, L. Wang et al., "DNA methylation analysis of the SHOX2 and RASSF1A panel in bronchoalveolar lavage fluid for lung cancer diagnosis," Journal of Cancer, vol. 8, no. 17, pp. 3585-3591, 2017.

[46] Q. Feng, S. E. Hawes, J. E. Stern et al., "DNA methylation in tumor and matched normal tissues from non-small cell lung cancer patients," Cancer Epidemiology Biomarkers \& Prevention, vol. 17, no. 3, pp. 645-654, 2008.
[47] R.-h. Xu, W. Wei, M. Krawczyk et al., "Circulating tumour DNA methylation markers for diagnosis and prognosis of hepatocellular carcinoma," Nature Materials, vol. 16, no. 11, pp. 1155-1161, 2017.

[48] P. Pawlikowska, V. Faugeroux, M. Oulhen et al., "Circulating tumor cells (CTCs) for the noninvasive monitoring and personalization of non-small cell lung cancer (NSCLC) therapies," Journal of Thoracic Disease, vol. 11, no. S1, pp. S45-s56, 2019.

[49] G. Hamilton and B. Rath, "Mesenchymal-epithelial transition and circulating tumor cells in small cell lung cancer," Advances in Experimental Medicine and Biology, vol. 994, pp. 229-245, 2017.

[50] M. Umer, R. Vaidyanathan, N.-T. Nguyen, and M. J. A. Shiddiky, "Circulating tumor microemboli: progress in molecular understanding and enrichment technologies," Biotechnology Advances, vol. 36, no. 4, pp. 1367-1389, 2018.

[51] K. C. Andree, G. van Dalum, and L. W. M. M. Terstappen, "Challenges in circulating tumor cell detection by the CellSearch system," Molecular Oncology, vol. 10, no. 3, pp. 395407, 2016.

[52] L. Cabel, C. Proudhon, H. Gortais et al., "Circulating tumor cells: clinical validity and utility," International Journal of Clinical Oncology, vol. 22, no. 3, pp. 421-430, 2017.

[53] H. I. Scher, R. P. Graf, N. A. Schreiber et al., "Assessment of the validity of nuclear-localized androgen receptor splice variant 7 in circulating tumor cells as a predictive biomarker for castration-resistant prostate cancer," JAMA Oncology, vol. 4, no. 9, pp. 1179-1186, 2018.

[54] H. Shao, H. Im, C. M. Castro, X. Breakefield, R. Weissleder, and H. Lee, "New technologies for analysis of extracellular vesicles," Chemical Reviews, vol. 118, no. 4, pp. 1917-1950, 2018.

[55] S. Gholizadeh, M. Shehata Draz, M. Zarghooni et al., "Microfluidic approaches for isolation, detection, and characterization of extracellular vesicles: current status and future directions," Biosensors and Bioelectronics, vol. 91, pp. 588605, 2017.

[56] G. Chen, A. C. Huang, W. Zhang et al., "Exosomal PD-L1 contributes to immunosuppression and is associated with anti-PD-1 response," Nature, vol. 560, no. 7718, pp. 382-386, 2018.

[57] L. Li, W. Li, N. Chen et al., "FLI1 exonic circular RNAs as a novel oncogenic driver to promote tumor metastasis in small cell lung cancer," Clinical Cancer Research, vol. 25, no. 4, pp. 1302-1317, 2019.

[58] N. Chen, G. Zhao, X. Yan et al., "A novel FLI1 exonic circular RNA promotes metastasis in breast cancer by coordinately regulating TET1 and DNMT1," Genome Biology, vol. 19, no. 1, p. $218,2018$.

[59] J. Rodon, J.-C. Soria, R. Berger et al., "Genomic and transcriptomic profiling expands precision cancer medicine: the WINTHER trial," Nature Medicine, vol. 25, no. 5, pp. 751-758, 2019.

[60] A. K. Krug, D. Enderle, C. Karlovich et al., "Improved EGFR mutation detection using combined exosomal RNA and circulating tumor DNA in NSCLC patient plasma," Annals of Oncology, vol. 29, no. 3, pp. 700-706, 2018.

[61] J. D. Merker, G. R. Oxnard, C. Compton et al., "Circulating tumor DNA analysis in patients with cancer: American Society of Clinical Oncology and College of American Pathologists joint review," Journal of Clinical Oncology, vol. 36, no. 16, pp. 1631-1641, 2018. 
[62] A. Stewart, E. A. Coker, S. Pölsterl et al., "Differences in signaling patterns on PI3K inhibition reveal context specificity in KRAS-mutant cancers," Molecular Cancer Therapeutics, vol. 18, no. 8, pp. 1396-1404, 2019.

[63] J. K. Sicklick, S. Kato, R. Okamura et al., "Molecular profiling of cancer patients enables personalized combination therapy: the I-PREDICT study," Nature Medicine, vol. 25, no. 5, pp. 744-750, 2019. 